\title{
Synthesis of two-dimensional antenna arrays by the method of generalized separation of variables
}

\author{
Yaroshko S. M. ${ }^{1}$, Yaroshko S. A. ${ }^{2}$ \\ ${ }^{1}$ Lviv Polytechnic National University, \\ 12 S. Bandera Str., 79013, Lviv, Ukraine \\ ${ }^{2}$ Ivan Franko National University of Lviv, \\ 1 Universytetska Str., 79000, Lviv, Ukraine
}

(Received 7 October 2019; Revised 9 November 2019; Accepted 10 November 2019)

\begin{abstract}
The method of generalized separation of variables is used to solve the problem of synthesis of antenna array with given form according to a given amplitude radiation pattern. An approximate solution obtained as a sum of terms with separated indexes. Such terms are calculated successively one by one at each step of the method from the condition of minimum of the corresponding functional. The minimization problem is reduced to the problem of solving the system of non-linear algebraic equations. General approaches of the method are specified for the cases of flat rectangular, flat hexagonal and cylindrical arrays. Proper attention has been paid to building effective computing procedures. The numerical results of solving several model problems are given. It is shown that the method allows us to get a satisfactory solution from a practical point of view in two to three steps.
\end{abstract}

Keywords: separation of variables, synthesis of the antenna array, amplitude radiation pattern, non-linear inverse problem.

2000 MSC: $65 \mathrm{C} 20$

UDC: $519.677,004.42$

DOI: $10.23939 / \mathrm{mmc} 2019.02 .386$

\section{Variational formulation of the synthesis problem}

The direct problem of the theory of radiation systems is to determine the behaviour $f(\varphi, \vartheta)$ of the electromagnetic field at infinity (this property is called the radiant pattern, RP) by the given distribution $U(x, y)$ of current at the surface of a continuous antenna, of by the discrete distribution $U=\left(u_{n m}\right)_{n=1}^{N} M_{m=1}^{M}$ of current at the radiators of antenna array. The solution of this problem is known [1]. It can be written in the form

$$
f=A U,
$$

where $A$ is a linear bounded operator, the specific of which depends on the type of antenna: for the continuously opened antenna this is an integral operator, while for the antenna grid there is a so-called summatory operator.

The classic formulation of the inverse problem (synthesis problem) consists in solving the equation (1). In other words it consists in finding the distribution of current $U$ by the known pattern $f$. It is known [2] that such problem is ill-posed. Firstly, it is exact solution exists only for a limited class of patterns. Secondly, there is no continuous dependency of a solution from the right-hand side part of the equation. That is why at practice, instead of the problem (1) another problem is considered [1,3] the one of minimization of the functional

$$
\sigma=\|A U-f\|_{f}^{2}+t\|U\|_{u}^{2} .
$$

Here, the second operand with the weight factor $t \geqslant 0$ is used for the regularization of the problem. Later on, it will be explained how the norms can be introduced. 
Often in practice, it is interesting to know only the distribution by the corner direction of energy, radiated by the antenna. That is, the absolute value of function $f(\varphi, \vartheta)$.

$$
f(\varphi, \vartheta)=F(\varphi, \vartheta) \times \exp (\arg f(\varphi, \vartheta))
$$

Note, that no restriction is put on the phase $\mathrm{RP} \exp (\arg f(\varphi, \vartheta))$. In this case, the following equation should be solved

$$
|A U|=F,
$$

where $F=F(\varphi, \vartheta)$ is a non-negative real bounded function of two variables, which is defined at the observation region $\Omega$. The function $F$ is a given amplitude RP (ARP). The region $\Omega$ is a corporeal angle, whose value and orientation depends on a specific problem.

The problem (3) is much more complex than (1). For the same reasons, as the previous one, this problem is ill-posed. Besides, it is non-linear and might have multiple solutions. To find some approximate solution to the problem (3), the minimization problem of the functional is considered $[1,3]$

$$
\sigma=\||A U|-F\|_{f}^{2}+t\|U\|_{u}^{2} .
$$

The functional (4) is non-convex [4]. It can have multiple local extremums, including the ones with equal values. From a practical point of view, it is convenient that there exist multiple solutions, because it gives a possibility to choose one of them, for example such, which is the easiest one to implement technically. Note, that the question of finding the global minimum of functional (4), identification of the number of solutions, analysis of their branching, goes out of the scope of this article.

\section{Generalized separation of variables}

Finding the point of a minimum of the functionals (2), (4) is linked with major computational complexities, because it implies a function of two variables, or a matrix of large dimensions. To decrease the computational complexity, the approximate solution will be queried as a sum of terms with separated variables [5] (see also [1,3]). The number of such terms is not fixed. They are retrieved sequentially: the next summand (given that all the previous ones are known) should minimize the functional. Every new term improves the accuracy of the desired solution and minimizes the value of the initial functional. The computation can be stopped at any moment, for example, when the synthesized RP approximates the initial pattern strong enough. Such an approach has been used in [6,7] for solving the multidimensional integral equations. Note, that the generalization method with the sequential calculation of terms is different from the method, described in [8].

Let us consider the synthesis problems of two-dimensional conformal antenna array by the given ARP. The summatory operator $A$ acts from the Euclid space $H_{u}=\mathbb{C}^{N \times M}$ complex-valued matrices to the Hilbert space $H_{f}=L_{2}[\Omega]$ of complex-valued functions of two arguments.

In the space $H_{f}$, the scalar product and the norm are defined [1]:

$$
\left(f_{1}, f_{2}\right)_{f}=\iint_{\Omega} f_{1}(\varphi, \vartheta) f_{2}^{*}(\varphi, \vartheta) p(\varphi, \vartheta) d \Omega, \quad\|f\|_{f}^{2}=(f, f)_{f}
$$

where $p(\varphi, \vartheta) \geqslant 0$ is the weight function.

In the space $H_{u}$ the scalar product is defined:

$$
(a, b)_{u}=\frac{1}{N M} \sum_{n=1}^{N} \sum_{m=1}^{M} a_{n m} b_{n m}^{*}
$$

where $a=\left\{a_{n m}\right\}, b=\left\{a_{n m}\right\}$ are matrices; the symbol "** denotes the complex conjugation. 
Let us denote

$$
\langle a, b\rangle_{1}=\frac{1}{N} \sum_{n=1}^{N} a_{n m} b_{n m}^{*} ; \quad\langle a, b\rangle_{2}=\frac{1}{M} \sum_{m=1}^{M} a_{n m} b_{n m}^{*} .
$$

Then the equality $(a, b)_{u}=\left\langle\langle a, b\rangle_{1}\right\rangle_{2}=\left\langle\langle a, b\rangle_{2}\right\rangle_{1}$ is fulfilled, where the denotation $\left\langle\langle\cdot, \cdot\rangle_{1}\right\rangle_{2}$ means the sequential summation by the corresponding indexes.

The approximate solution will be queried in the form [5]

$$
U^{(s)}=\sum_{j=1}^{s} \bar{v}^{(j) T} \bar{w}^{(j)}=U^{(s-1)}+\bar{v}^{(s) T} \bar{w}^{(s)}, \quad s=1,2, \ldots,
$$

where $U^{(s)}$ is the distribution matrix of current at the radiators of array, $T$ is the symbol of vector transposition.

At the first step of the method $(s=1)$ let us assume that $U^{(0)} \equiv 0$. The summands in $(8)$ are found successively from the condition of minimum of the corresponding functional. At the $s$-th step, provided that the distribution of current $U^{(s-1)}$ is known, let us find the corrective $\bar{v}^{(s) T} \bar{w}^{(s)}$ which minimizes the functional

$$
\sigma_{s}=\left\|\left|A U^{(s)}\right|-F\right\|_{f}^{2}+t\left\|U^{(s)}\right\|_{u}^{2} .
$$

Let us put the partial variations of the functional (9) on the variables $\bar{v}^{(s)}$ and $\bar{w}^{(s)}$. Thus, it's easy to obtain a system of Euler's equations of the functional (9), where $A^{*}$ is the operator adjoint to $A$ :

$$
\left\{\begin{array}{l}
\left\langle\left(A^{*}\left(A U^{(s)}-F \exp \left[i \arg A U^{(s)}\right]\right)+t U^{(s)}, \bar{w}^{(s)}\right\rangle_{2}=0,\right. \\
\left\langle\left(A^{*}\left(A U^{(s)}-F \exp \left[i \arg A U^{(s)}\right]\right)+t U^{(s)}, \bar{v}^{(s)}\right\rangle_{1}=0 .\right.
\end{array}\right.
$$

Let us denote $A U^{(s)}=f^{(s)}$, in the system (10) move to the right-hand side the known additions of the solution and the phase RP, and we will get

$$
\left\{\begin{array}{l}
\left\langle\left(A^{*} A+t I\right) \bar{v}^{(s) T} \bar{w}^{(s)}, \bar{w}^{(s)}\right\rangle_{2}=\left\langle A^{*}\left(F \exp \left[i \arg f^{(s)}\right]-f^{(s-1)}\right)-t U^{(s-1)}, \bar{w}^{(s)}\right\rangle_{2}, \\
\left\langle\left(A^{*} A+t I\right) \bar{v}^{(s) T} \bar{w}^{(s)}, \bar{v}^{(s)}\right\rangle_{1}=\left\langle A^{*}\left(F \exp \left[i \arg f^{(s)}\right]-f^{(s-1)}\right)-t U^{(s-1)}, \bar{v}^{(s)}\right\rangle_{1} .
\end{array}\right.
$$

\subsection{The method of successive approximations}

The system (11) is non-linear. Moreover, the phase pattern at the right-hand side of the system depends on the unknown $\bar{v}^{(s)}, \bar{w}^{(s)}$. However, the left-hand side of the first system equation is linear with respect to $\bar{v}^{(s)}$, the left-hand side of the second equation is linear with respect to $\bar{w}^{(s)}$. In order to solve it the method of successive approximations can be applied. At each step of this method the equations are being solved as linear in respect to a corresponding unknown and via the value of the unknown from the previous step. To build the right-hand side parts of the system the initial approximations of the unknowns are being used. The iteration process of this method can be written as follows:

$$
\left\{\begin{array}{l}
\left\langle\left(A^{*} A+t I\right) \bar{v}^{(s, j) T} \bar{w}^{(s, j-1)}, \bar{w}^{(s, j-1)}\right\rangle_{2} \\
\quad=\left\langle A^{*}\left(F \exp \left[i \arg f^{(s, j-1, j-1)}\right]-f^{(s-1)}\right)-t U^{(s-1)}, \bar{w}^{(s, j-1)}\right\rangle_{2}, \\
\quad\left\langle\left(A^{*} A+t I\right) \bar{v}^{(s, j) T} \bar{w}^{(s, j)}, \bar{v}^{(s, j)}\right\rangle_{1} \\
\quad=\left\langle A^{*}\left(F \exp \left[i \arg f^{(s, j, j-1)}\right]-f^{(s-1)}\right)-t U^{(s-1)}, \bar{v}^{(s, j)}\right\rangle_{1}, \quad j=1,2, \ldots,
\end{array}\right.
$$

where the denotation $f^{(s, j, j-1)}=A\left(U^{(s-1)}+\bar{v}^{(s, j) T} \bar{w}^{(s, j-1)}\right)$ is used; and $\bar{v}^{(s, j)}, \bar{w}^{(s, j)}$ are the $j$-ths approximations of the queried unknowns; $\bar{v}^{(s, 0)}, \bar{w}^{(s, 0)}$ are some initial approximations. It is easy to show that at each step the process (12) diminishes the value of the non-negative functional (9) and, thus, the sequence of the functional's values converges. 
At each step of the iteration process, the sub-systems of the system (11) are being solved with the changed right-hand sides. This is equal to replacing the functional (4) with the convex functionals

$$
\begin{aligned}
& \sigma_{j}^{(1)}(U)=\left\|F \exp \left[i \arg f^{(s, j-1, j-1)}\right]-A U\right\|_{f}^{2}+t\|U\|_{u}^{2}, \\
& \sigma_{j}^{(2)}(U)=\left\|F \exp \left[i \arg f^{(s, j, j-1)}\right]-A U\right\|_{f}^{2}+t\|U\|_{u}^{2} .
\end{aligned}
$$

It can be easily seen that for any continuous functions: real non-negative $F$, real $\psi$ and complex $f$ the following inequality is fulfilled

$$
\sigma \leqslant \sigma_{\psi},
$$

where $\sigma=\|F-|f|\|_{f}^{2}, \sigma_{\psi}=\|F \exp (i \psi)-f\|_{f}^{2}$.

Indeed, it is true that $\left(\sigma_{\psi}-\sigma\right) / 2=-\operatorname{Re}(F \exp (i \psi-i \arg f), f)=(F(1-\cos (\psi-\arg f)),|f|) \geqslant 0$.

After the $(j-1)$-th step of the iteration process the current distribution is known $U^{(s, j-1, j-1)}=$ $U^{(s-1)}+\bar{v}^{(s, j-1) T} \bar{w}^{(s, j-1)}$. It is obvious that $\sigma\left(U^{(s, j-1, j-1)}\right)=\sigma_{j}^{(1)}\left(U^{(s, j-1, j-1)}\right)$. After having solved the first sub-system in (12) we get $U^{(s, j, j-1)}$, and $\sigma_{j}^{(1)}\left(U^{(s, j, j-1)}\right)<\sigma_{j}^{(1)}\left(U^{(s, j-1, j-1)}\right)$. But, according to $(13), \sigma\left(U^{(s, j, j-1)}\right) \leqslant \sigma_{j}^{(1)}\left(U^{(s, j, j-1)}\right)$, that's why

$$
\sigma\left(U^{(s, j, j-1)}\right)<\sigma\left(U^{(s, j-1, j-1)}\right) .
$$

Similarly, $\sigma\left(U^{(s, j, j-1)}\right)=\sigma_{j}^{(2)}\left(U^{(s, j, j-1)}\right)$. After the second sub-system in (12) is solved, we get $\sigma_{j}^{(2)}\left(U^{(s, j, j)}\right)<\sigma_{j}^{(2)}\left(U^{(s, j, j-1)}\right)$. According to $(13), \sigma\left(U^{(s, j, j)}\right) \leqslant \sigma_{j}^{(2)}\left(U^{(s, j, j)}\right)$. Thus

$$
\sigma\left(U^{(s, j, j)}\right)<\sigma\left(U^{(s, j, j-1)}\right) .
$$

From (14) and (15) it follows that at every step of the iteration process (12) the value $\sigma_{s}$ is monotonously ascending. The presented considerations do not depend on the value $s$, so they are true for every step of the method of generalized division of variables.

\subsection{Choosing the initial approximation}

When solving the system (11) (or (12)) it is important to remember that it has an incident trivial solution $\bar{v}^{(s)} \equiv 0, \bar{w}^{(s)} \equiv 0$, at which the functional (9) does not reach the minimum [1]. Due to the fact that at each step of the method of successive approximations the value of the functional does not grow, in order to prevent the iteration process from giving the trivial solution, the initial approximation of the $s$-th addition must be chosen so, that the value $\sigma_{s}$ on it is less than $\sigma_{s}\left(U^{(s-1)}\right)$.

Let us choose any initial distribution of current in the form

$$
c \bar{v}^{(s, 0) T} \bar{w}^{(s, 0)}=c u^{(s)},
$$

where $c$ is a not-defined complex-valued coefficient. Let us now consider the functional $\sigma_{s}$ as a function of a complex argument $c$ and find such value $c_{s}$, that $\sigma_{s}\left(c_{s}\right)<\sigma_{s}(0)$,

$$
\sigma_{s}(c)=\left\|\left|f^{(s-1)}+c A u^{(s)}\right|-F\right\|_{f}^{2}+t\left\|U^{(s-1)}+c u^{(s)}\right\|_{u}^{2},
$$

Let us consider an auxiliary functional

$$
\sigma_{s}^{(3)}(c)=\left\|F \exp \left[i \arg f^{(s-1)}\right]-\left(f^{(s-1)}+c A u^{(s)}\right)\right\|_{f}^{2}+t\left\|U^{(s-1)}+c u^{(s)}\right\|_{u}^{2} .
$$

According to (13) it is fulfilled $\sigma_{s} \leqslant \sigma_{s}^{(3)}$. So, let us find a value $c_{s}$ such, that $\sigma_{s}^{(3)}$ reaches the minimum. It is easy to receive

$$
c_{s}=\frac{\left(F \exp \left[i \arg f^{(s-1)}\right]-f^{(s-1)}, A u^{(s)}\right)_{f}-t\left(U^{(s-1)}, u^{(s)}\right)_{u}}{\left\|A u^{(s)}\right\|_{f}^{2}+t\left\|u^{(s)}\right\|_{u}^{2}} .
$$


For the coefficient $c_{s}$, calculated by the formula (18), the relation is true $\sigma_{s}(0)=\sigma_{s}^{(3)}(0)>\sigma_{s}^{(3)}\left(c_{s}\right) \geqslant$ $\sigma_{s}\left(c_{s}\right)$. Thus, the distribution of current $c_{s} u_{s}$ can be taken as the initial approximation in the iteration process (12).

Note, that to find the first term $\bar{v}^{(1) T} \bar{w}^{(1)}$ the optimization coefficient $c_{1}$ can be omitted. Indeed, at this stage the radiant pattern in the right-hand side of the system (12) does not depend on $c_{1}$, and to build the left-hand side of the first sub-system, only the initial approximation of the vector $\bar{w}^{(1)}$ is used. Thereby, the unknown coefficient $c_{1}$ can be moved to the wanted vector $\bar{v}^{(1)}$.

\subsection{Finiteness of the method's algorithm}

In the synthesis of antennas grids, it is possible to calculate the distribution of current, at which the initial functional reaches the minimum, in a finite number of steps. For this, the computational scheme, described below, should be slightly modified. In order to prove this statement it is enough to demonstrate that the matrices $\bar{v}^{(j) T} \bar{w}^{(1)}, j=1, \ldots, N \times M$ form the base in the $(N \times M)$-dimensional matrix space, and to indicate the way of choosing the matrices coefficients such, that ensure reaching the minimum.

The first two terms in (8) are linearly independent due to their computing method. Otherwise, they would be proportional to each other and we would obtain the distribution of current $U^{(2)}=k U^{(1)}$ such, that would increase the value of the functional, instead of minimizing it.

When finding the next summands, let us put the distribution of current $U^{(s)}$, differently to (8), in the form

$$
U^{(s)}=\sum_{j=1}^{s-1} a_{j}^{(s)} \bar{v}^{(j) T} \bar{w}^{(j)}+\bar{v}^{(s) T} \bar{w}^{(s)}=U^{(s-1)}+\bar{v}^{(s) T} \bar{w}^{(s)}, \quad s=3,4, \ldots
$$

The unknown vectors $\bar{v}^{(s)}, \bar{w}^{(s)}$ and the coefficients $a_{j}^{(s)}, j=1, \ldots, s-1$ are obtained from the minimum condition of the same functional (9).

As already explained, the minimization problem of $\sigma_{s}$ can be transformed into the problem of solving the system of Euler equations. This system will contain three sub-systems: the first two identical to the ones in the system (11), where the distribution of current (8) is changed to (19); the third sub-system is formulated as

$$
\begin{aligned}
& \left(\left(A^{*} A+t I\right) U^{(s-1)}, \bar{v}^{(j) T} \bar{w}^{(j)}\right)_{u} \\
& \quad=\left(A^{*}\left(F \exp \left[i \arg f^{(s)}\right]-A \bar{v}^{(s) T} \bar{w}^{(s)}\right)-t \bar{v}^{(s) T} \bar{w}^{(s)}, \bar{v}^{(j) T} \bar{w}^{(j)}\right)_{u}, \quad j=1, \ldots, s-1 .
\end{aligned}
$$

The obtained system possesses the same properties, as (11): the left-hand side of each subsystem is linear relative to the corresponding unknowns. Thus, it can be solved using the method of successive approximations, which has been presented earlier. It can be proved that such process at every step decreases the value of the initial functional.

Thereby, every new calculated term in (19) will be linearly independent of all the preceding ones. At the last $(N \times M)$-th step the solution will be queried in the form of distribution at the obtained basis of matrix space with free coefficients, which ensures the completion of the computational process at this step. The obtained solution is at least a stationary point of the functional (9). If the initial approximations are chosen properly at the intermediate steps, the considered functional will achieve its minimum at the calculated solution.

The described computational scheme serves only for the proof of the method algorithm's finiteness. For the practical reasons, it is usually enough to calculate $2-3$ additions in (8) or in (19). Note, that the first two additions of both options coincide.

\section{Synthesis of conformal antenna arrays}

Let us specify how to solve the synthesis problem of the antenna array of a given form by using the method of generalized separation of variables for certain type of antennas: flat rectangular and 
hexagonal and cylindrical arrays. Let us also present some numeric results. Of course, there are many known solutions of synthesis problems of such antennas. For instance, rectangular arrays were considered also in $[4,9,10]$, hexagonal - in $[11,12]$. We can see results of the synthesis of the cylindrical array in $[1,3]$. Nevertheless, our main goal is to show the method of generalized separation works in specific cases and to demonstrate its effectiveness. All numerical results are new.

\subsection{Flat rectangular antennas}

Consider a flat array, which consists of $(N \times M)$ linearly polarized radiators, situated in the nodes of the rectangular grid, which is positioned in parallel to the axis of the Cartesian coordinates system $(X O Y)$. RP of such array has the form [13]

$$
f(\varphi, \vartheta)=A U \equiv \sum_{n=1}^{M} \sum_{m=1}^{M} u_{n m} f_{n m}(\varphi, \vartheta) \exp \left[i k \sin \vartheta\left(x_{n} \cos \varphi+y_{m} \sin \varphi\right)\right]
$$

where $u_{n m}$ is the current at the radiator with indexes $(n, m) ; f_{n m}(\varphi, \vartheta)$ is the RP of this radiator at the unite current (in order to take into account the mutual influence this pattern is measured under the condition that the rest of passive radiators are present); $k$ is wave number; $\left(x_{n}, y_{m}\right)$ are Cartesian coordinates of $n m$-th radiator; $\varphi \in[0 ; 2 \pi], \vartheta \in[0 ; \pi / 2]$, and the upper hemisphere $\Omega$ is the angle of observation.

As in [5] the functional (9) is then transformed into

$$
\sigma_{s}=\int_{0}^{2 \pi} \int_{0}^{\pi / 2}\left(\left|f^{(s)}(\varphi, \vartheta)\right|-F(\varphi, \vartheta)\right)^{2} p(\varphi, \vartheta) \sin \vartheta d \vartheta d \varphi+\frac{t}{N M} \sum_{n=1}^{N} \sum_{m=1}^{M}\left|u_{n m}^{(s)}\right|^{2},
$$

and the system (11) is expressed as

$$
\begin{aligned}
& \sum_{n^{\prime}=1}^{N} v_{n^{\prime}}^{(s)} \sum_{m=1}^{M} w_{m}^{(s) *} \sum_{m^{\prime}=1}^{M} w_{m^{\prime}}^{(s)} D_{n m}^{n^{\prime} m^{\prime}}+\frac{t}{N M} v_{n}^{(s)} \sum_{m=1}^{M}\left|w_{m}^{(s)}\right|^{2} \\
& \quad=\sum_{m=1}^{M} w_{m}^{(s) *}\left(C_{n m}\left[F \exp \left[i \arg f^{(s)}-f^{(s-1)}\right]\right]-\frac{t}{N M} u_{n m}^{(s-1)}\right), \quad n=1, \ldots, N, \\
& \sum_{m^{\prime}=1}^{M} w_{m^{\prime}}^{(s)} \sum_{n=1}^{N} v_{n}^{(s) *} \sum_{n^{\prime}=1}^{N} v_{n^{\prime}}^{(s)} D_{n m}^{n^{\prime} m^{\prime}}+\frac{t}{N M} w_{m}^{(s)} \sum_{v=1}^{N}\left|v_{n}^{(s)}\right|^{2} \\
& \quad=\sum_{n=1}^{N} v_{n}^{(s) *}\left(C_{n m}\left[F \exp \left[i \arg f^{(s)}-f^{(s-1)}\right]\right]-\frac{t}{N M} u_{n m}^{(s-1)}\right), \quad m=1, \ldots, M,
\end{aligned}
$$

where the following denotation is used

$$
\begin{aligned}
C_{n m}[\Phi]= & \int_{0}^{2 \pi} \int_{0}^{\pi / 2} \Phi(\varphi, \vartheta) f_{n m}^{*}(\varphi, \vartheta) p(\varphi, \vartheta) \exp \left[-i k \sin \vartheta\left(x_{n} \cos \varphi+y_{m} \sin \varphi\right)\right] \sin \vartheta d \vartheta d \varphi \\
D_{n m}^{n^{\prime} m^{\prime}}= & \int_{0}^{2 \pi} \int_{0}^{\pi / 2} f_{n^{\prime} m^{\prime}}(\varphi, \vartheta) f_{n m}^{*}(\varphi, \vartheta) p(\varphi, \vartheta) \\
& \times \exp \left[i k \sin \vartheta\left(\left(x_{n^{\prime}}-x_{n}\right) \cos \varphi+\left(y_{m^{\prime}}-y_{m}\right) \sin \varphi\right)\right] \sin \vartheta d \vartheta d \varphi
\end{aligned}
$$

The weight function $p(\varphi, \vartheta)$ can be used to improve the approximation of a given RP in certain range of angles.

Quite often in practice, the diagrams $f_{n m}$ of the radiators are considered to be the same. Then they can be moved out of the summation sign in (20). The remaining sum is called the grid multiplier. If $f_{n m}(\varphi, \vartheta) \equiv 1$, then the RP of the antenna is equal to the grid multiplier. Let us analyze this case 
and demonstrate that the integrals (24) can be calculated analytically if the simplest weight function $p(\varphi, \vartheta) \equiv$ const is used and moved out of the integral.


Fig. 1. Given and synthesized ARP of Problem 1.

Let us denote $\Delta_{n m}^{n^{\prime} m^{\prime}}=\sqrt{\left(x_{n^{\prime}}-x_{n}\right)^{2}+\left(y_{m^{\prime}}-y_{m}\right)^{2}}, \frac{x_{n}-x_{n n^{\prime}}}{\Delta_{n m}^{n^{\prime} m^{\prime}}}=\sin \alpha_{n m}^{n^{\prime} m^{\prime}}, \frac{y_{m}-y_{m^{\prime}}}{\Delta_{n m}^{n^{\prime} m^{\prime}}}=\cos \alpha_{n m}^{n^{\prime} m^{\prime}}$. Then

$$
\begin{aligned}
D_{n m}^{n^{\prime} m^{\prime}} & =\int_{0}^{2 \pi} \int_{0}^{\pi / 2} \exp \left[i k \Delta_{n m}^{n^{\prime} m^{\prime}} \sin \vartheta \sin \left(\varphi+\alpha_{n m}^{n^{\prime} m^{\prime}}\right)\right] \sin \vartheta d \vartheta d \varphi \\
& =\int_{0}^{\pi} \int_{0}^{\pi / 2} \cos \left(i k \Delta_{n m}^{n^{\prime} m^{\prime}} \sin \vartheta \sin \varphi\right) \sin \vartheta d \vartheta d \varphi=2 \pi \int_{0}^{\pi / 2} J_{0}\left(k \Delta_{n m}^{n^{\prime} m^{\prime}} \sin \vartheta\right) \sin \vartheta d \vartheta \\
& =2 \pi \sqrt{\frac{\pi}{2}} J_{1 / 2}\left(k \Delta_{n m}^{n^{\prime} m^{\prime}}\right) / \sqrt{k \Delta_{n m}^{n^{\prime} m^{\prime}}}=2 \pi \sin \left(k \Delta_{n m}^{n^{\prime} m^{\prime}}\right) /\left(k \Delta_{n m}^{n^{\prime} m^{\prime}}\right), \quad n \neq n^{\prime} \vee m \neq m^{\prime} .
\end{aligned}
$$

In case $n=n^{\prime}, m=m^{\prime}$, then $\Delta_{n m}^{n^{\prime} m^{\prime}}=0$, and $D_{n m}^{n m}=2 \pi$ can be easily calculated.

\section{Problem 1.}

Let us give an example of solving the synthesis problem for a specific array, build from $21 \times 21$ identical radiators. Their coordinates are defined by the formulas $x_{n}=\left(n-n_{c}\right) \lambda, y_{m}=\left(y-y_{c}\right) \lambda$, where $\lambda$ is the wave length, $\left(n_{c} ; m_{c}\right)=(11 ; 11)$ are the indices of the central radiator, located at the beginning of the coordinates system. The following ARP is given

$$
F(\varphi, \vartheta)= \begin{cases}\cos ^{2}(\vartheta \sqrt{5-4 \sin 5 \varphi}), & -0.1 \pi<\varphi<0.7 \pi, 0 \leqslant \vartheta \leqslant \frac{\pi}{2 \sqrt{5-4 \sin 5 \varphi}} \\ \cos ^{2} 3 \vartheta, & 0.7 \pi \leqslant \varphi \leqslant 1.9 \pi, 0 \leqslant \vartheta<\frac{\pi}{6} \\ 0, & \text { in other cases. }\end{cases}
$$


The solution has been queried in form (8), the functional (21) has been minimized, and the weight function $p(\varphi, \vartheta)=$ const has been chosen so, that $\|F\|_{f}^{2}=1$. The computation process has lasted until $\sigma_{s-1}-\sigma_{s} \leqslant \varepsilon$.

To achieve the accuracy $\varepsilon=10^{-4}$ seven summands in the matrix of distribution of current have been calculated. The corresponding values of the functional $(21)$ are: $\sigma_{1}=0.14033, \sigma_{2}=0.03879$, $\sigma_{3}=0.01439, \sigma_{4}=0.00541, \sigma_{5}=0.00389, \sigma_{6}=0.00274, \sigma_{7}=0.00215$. Figure 1 contains isolines of the given $F$ and several charts of $F$ and the synthesized $\operatorname{ARP}\left|f^{(1)}\right|,\left|f^{(2)}\right|,\left|f^{(3)}\right|,\left|f^{(4)}\right|$ at certain fixed values of $\varphi$.

In this problem it is not enough to use only the first term in the obtained solution. However, already the first three terms provide quite an accurate approximation to the given pattern (25).

\subsection{Flat hexagonal antennas}

Another common type of flat AG is a hexagonal (triangular) array. It usually consists of identical radiators, located in the nodes of a regular triangular grid. With proper indexing of the radiators, the described method can be used to solve the synthesis problems of the hexagonal antenna.


Fig. 2. Hexagonal antenna array: scheme of radiators location (on the left); level lines of the synthesized amplitude patterns of Problem 2 (on the right).

Let us assign to every radiator a pair of indexes $(n, m)$, as it is shown at the figure 2. Let's assume that $N=M=2 n_{c}-1$, where $\left(n_{c}, n_{c}\right)$ are the indexes of the central grid radiator, located at the beginning of the Cartesian coordinates system. Thus, the coordinates of the radiators can be defined by the formulas

$$
x_{n}=\left(\left(n-n_{c}\right)+\left(m-m_{c}\right) / 2\right) \frac{\lambda}{2}, \quad y_{m}=\frac{\sqrt{3}}{2}\left(m-m_{c}\right) \frac{\lambda}{2},
$$

where $\lambda$ is the length of the wave.

It can be easily seen that the obtained array does not contain the radiators with indexes $(n, m)$, such that $2|(n+m) /(N+1)-1| \geqslant 1$. Therefore, let us introduce the following denotations

$$
\tilde{f}_{n m}(\varphi, \vartheta)= \begin{cases}f_{n m}(\varphi, \vartheta), & 2|(n+m) /(N+1)-1|<1 \\ 0, & \text { otherwise }\end{cases}
$$

If in the formula (20) the $f_{n m}$ is replaced by $\tilde{f}_{n m}$, then it can be used to compute the RP of the hexagonal array. 
Similarly, to the previous case, let us query the solution of the synthesis problem (3) in the form (8), as the point of a minimum of the functional (9) (or (21)). For this purpose let us use the system (11), where $C_{n m}[\Phi]$ and $D_{n m}^{n^{\prime} m^{\prime}}$ are being replaced by $\tilde{C}_{n m}[\Phi]$ and $\tilde{D}_{n m}^{n^{\prime} m^{\prime}}$ respectively:

$$
\begin{gathered}
\tilde{C}_{n m}[\Phi]= \begin{cases}C_{n m}[\Phi], & 2|(n+m) /(N+1)-1|<1 ; \\
0, & \text { otherwise. }\end{cases} \\
\tilde{D}_{n m}^{n^{\prime} m^{\prime}}= \begin{cases}D_{n m}^{n^{\prime} m^{\prime}}, & 2=|(n+m) /(N+1)-1|<1,2\left|\left(n^{\prime}+m^{\prime}\right) /(N+1)-1\right|<1 ; \\
0, & \text { otherwise. }\end{cases}
\end{gathered}
$$

To solve the obtained system, let us use the described method of successive approximations. All the finiteness conclusions, presented earlier, are valid in this case as well.

The integrals in (29) can be calculated analytically by using the same formulas, as in the previous cases, with just having replaced the definition $\Delta_{n m}^{n^{\prime} m^{\prime}}=\sqrt{\left(n^{\prime}-n\right)^{2}+\left(n^{\prime}-n\right)\left(m^{\prime}-m\right)=\left(m^{\prime}-m\right)^{2}}$.

\section{Problem 2.}

As a result of the modifications that have been made, an array has been synthesized for $N=15$, which implements a narrow ARP

$$
F(\varphi, \vartheta)= \begin{cases}\cos ^{2}(5 \vartheta), & 0 \leqslant \vartheta \leqslant \frac{\pi}{10} \\ 0, & \text { in other cases }\end{cases}
$$

To reach the accuracy $\varepsilon=10^{-4}$ four steps of the method have been made. The value of the minimized functional (21) at these steps are the following: $\sigma_{1}=0.0742, \sigma_{2}=0.0034, \sigma_{3}=0.00081$, $\sigma_{4}=0.00043$. Note, that already the second approximation of the given pattern (30) is quite accurate. It can be seen in Figure 2, that $\left|f^{(4)}\right|$ only decreases the values of the side chart petals.

\subsection{Cylindrical antennas}

A cylindrical array is a system of radiators, located at a cylindrical surface. Let it contain $N$ linear grids with $M$ radiators in each. The lines are located along the cylinder formation, $m$-ths radiators of each line belong to one surface and form a circular array. The radiant pattern of a cylindrical array with linearly polarized radiators can be presented as [13]

$$
f(\varphi, \vartheta)=A U \equiv \sum_{n=1}^{M} \sum_{m=1}^{M} u_{n m} f_{n m}(\varphi, \vartheta) \exp \left[i k\left(r \sin \vartheta \cos \left(\varphi-\varphi_{n}\right)+z_{m} \cos \vartheta\right)\right]
$$

where $k r$ is the electric radius of the cylinder; $\varphi_{n}$ is the axis coordinate of $n$-th line, $z_{m}$ is the vertical coordinate of $m$-th array circle in the cylindrical coordinates system; $\varphi \in[-\pi / 2 ; \pi / 2], \vartheta \in[0 ; \pi]$; and all the other denotations have the same meaning as in (20).

The solution of the problem (3) should be queried in the form (8). For this purpose the system (11) is being solved, with (23) and (24) being replaced by the following values respectively

$$
\begin{aligned}
C_{n m}[\Phi]= & \int_{-\pi / 2}^{\pi / 2} \int_{0}^{\pi} \Phi(\varphi, \vartheta) f_{n m}^{*}(\varphi, \vartheta) p(\varphi, \vartheta) \exp \left[-i k\left(r \sin \vartheta \cos \left(\varphi-\varphi_{n}\right)+z_{m} \cos \vartheta\right)\right] \sin \vartheta d \vartheta d \varphi \\
D_{n m}^{n^{\prime} m^{\prime}}= & \int_{-\pi / 2}^{\pi / 2} \int_{0}^{\pi} f_{n^{\prime} m^{\prime}}(\varphi, \vartheta) f_{n m}^{*}(\varphi, \vartheta) p(\varphi, \vartheta) \\
& \times \exp \left[i k\left(r \sin \vartheta\left(\cos \left(\varphi-\varphi_{n^{\prime}}\right)-\cos \left(\varphi-\varphi_{n}\right)\right)+\left(z_{m^{\prime}}-z_{m}\right) \cos \vartheta\right)\right] \sin \vartheta d \vartheta d \varphi
\end{aligned}
$$




\section{Problem 3.}

The cylindrical array consists of identical $9 \times 9$ radiators form the pattern like

$$
f_{n m}(\varphi, \vartheta)= \begin{cases}\sin ^{2} \vartheta \cos ^{2}\left(\varphi-\varphi_{n}\right), & \left|\varphi-\varphi_{n}\right|<\pi / 2, \\ 0, & \text { otherwise }\end{cases}
$$

where $\varphi_{n}=\left(n-n_{c}\right) \pi / k r,\left(n_{c}, m_{c}\right)$ are the indexes of the central radiator of the array.

The electric radius of the cylinder $k r=15\left(\Delta \varphi=15^{\circ}, \varphi_{9}-\varphi_{1}=96^{\circ}\right)$. The given ARP is a sum of three lobes of the form

$$
F^{(j)}(\varphi, \vartheta)= \begin{cases}\cos ^{2} 4 g^{(j)}(\varphi, \vartheta), & g^{(j)}(\varphi, \vartheta)<\pi / 8, \\ 0, & \text { in other cases }\end{cases}
$$

where $g^{(j)}(\varphi, \vartheta)=\arccos \left(\cos \left(\varphi-\varphi_{j}\right) \sin \left(\vartheta-\vartheta_{j}\right)\right)$, the angles $\left(\varphi_{j}, \vartheta_{j}\right)$ defines the direction of the pattern lobes: $\left(\varphi_{1}, \vartheta_{1}\right)=(-\pi / 8, \pi(6-\sqrt{3}) / 12),\left(\varphi_{2}, \vartheta_{2}\right)=(\pi / 8, \pi(6-\sqrt{3}) / 12)$ and $\left(\varphi_{3}, \vartheta_{3}\right)=$ $(0, \pi(6+\sqrt{3} / 2) / 12)$.
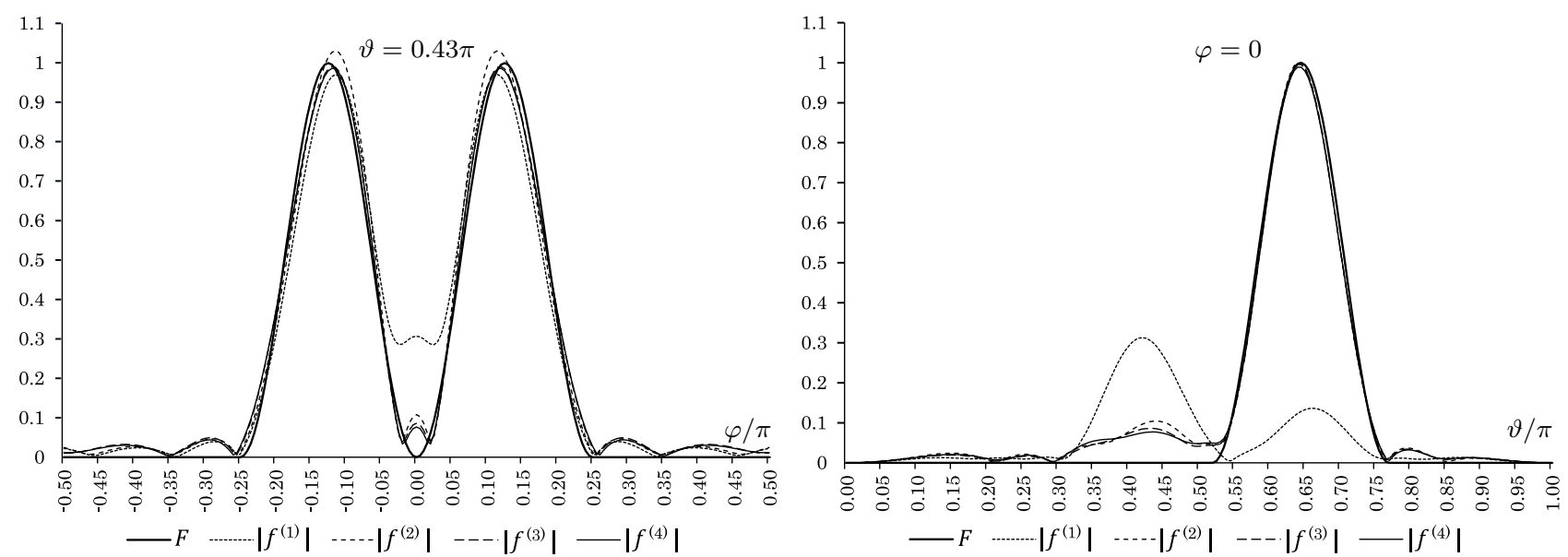

Fig. 3. Given and synthesized ARP in Problem 3.

Five terms of the approximate solution (8) have been calculated. The corresponding values of the functional are: $\sigma_{1}=0.28828, \sigma_{2}=0.01417, \sigma_{3}=0.01115, \sigma_{4}=0.01051, \sigma_{5}=0.01024$.

Problem 3 is significant for the method of generalized separation of variables, because the given ARP is such, that its variables can not be separated even approximately. Hence, the solution of the problem can not be presented with only one term of the decomposition (8) with proper accuracy. Indeed, after the first step of the method the value of the obtained functional remains quite big: $\sigma_{1} \approx 0.29\|F\|_{f}^{2}$. Figure 3 demonstrates that the pattern $\left|f^{(1)}\right|$ approximates only two out of three lobes of the given ARP, while $\left|f^{(2)}\right|$ approximates all lobes. The next summands of the solution improve this approximation and diminish the level of side pattern lobes.

\section{Conclusion}

The described method of generalized separation of variables (MGSV) is an effective tool for solving synthesis problems of two-dimensional antennas arrays. It uses a variational formulation of the problem and builds an approximate solution as a sum of terms with separated indexes. Every subsequent summand is calculated to make more precise the approximation when all previous ones are fixed. The method decreases the dimension of the problem since replace it by the system of one-dimensional nonlinear algebraic equations. The method of successive approximations can be used to solve this system. At each internal step of it, the proper linear system has to be solved. 
It is shown in the article that the described method of successive approximations is convergent so far as it diminishes the source functional at each own step. The new modification of the method of generalized separation of variables is described. It provides a way to obtain a stationary point of the minimized functional by the finite number of steps. The method for appreciable improving the computational efficiency of the MGSV is presented. We can fulfil a part of calculations analytically in many cases of synthesis a flat antenna array. It is specified how to use the MGSV to solve a synthesis problem with a hexagonal antenna array.

Several new model synthesis problems were solved by the MGSA for rectangular, hexagonal and cylindrical antennas arrays. It has been shown that the MGSA is equally effective in finding solutions for different given radiation patterns: narrow symmetric, unsymmetrical, many-lobes. In many cases, just the third term of an approximate solution reduces the level of error to about $1 \%$ of the norm of the prescribed pattern.

[1] Andrijchuk M. I., Vojtovich N. N., Savenko P. A., Tkachuk V. P. Sintez antenn po amplitudnoj diagramme napravlennosti. Chislennye metody i algoritmy. Kiev, Naukova dumka (1993), (in Russian).

[2] Tihonov A. N., Arsenin V. Ya. Metody resheniya nekorrektnyh zadach. Moskva, Nauka (1986), (in Russian).

[3] Bulatsyk O. O., Katsenelenbaum B. Z., Topolyuk Yu. P., Voitovich N. N. Phase Optimization Problems: Applications in Wave Field Theory. Weinheim, Wiley-VCH Verlag GmbH \& Co. KGaA (2010).

[4] Savenko P. O. Neliniini zadachi teorii syntezu vyprominiuiuchykh system z ploskym rozkryvom. Lviv, IPPMM NAN Ukrainy (2014), (in Ukrainian).

[5] Vojtovich N. N. Sintez dvumernoj antennoj reshetki s obobshhennym razdeleniem peremennyh. Radiotehnika i elektronika. 33 (12), 2637-2640 (1988), (in Russian).

[6] Voitovich M. M., Yaroshko S. A. A variational-iterative method for the generalized separation of variables in the solution of multidimensional integral equations. Journal of Mathematical Sciences. 96 (2), 3042-3046 (1999).

[7] Biletskyy V. M. Modification of a method of generalized separation of variables for the solution of multidimensional integral equations. Journal of Mathematical Sciences. 181 (3), 44-50 (2010).

[8] Kalenjuk P. I., Baraneckij Ja. E. Nitrebich Z. N. Obobshhennyj metod razdelenija peremennyh. Kiev, Naukova dumka (1993), (in Russian).

[9] Andriychuk M., Savenko P., Tkach M. Non-linear synthesis problems for plane radiating systems according to the prescribed power directivity pattern. Open Journal of Antennas and Propagation. 1 (2), 23-34 (2013).

[10] Protsakh L. P., Savenko P. O., Tkach M. D. About Branching of Solutions of Synthesis Problem for a Flat Aperture According to the Prescribed Amplitude Directivity Pattern. 2010 XVth International Seminar/Workshop on Direct and Inverse Problems of Electromagnetic and Acoustic Wave Theory (DIPED). 177-181 (2010).

[11] Andriichuk M.I. Syntez ploskoi heksahonalnoi antennoi reshitky za zadanoiu enerhetychnoiu diahramoiu spriamovanosti. Information Extraction and Proces. 38 (114), 5-12 (2013), (in Ukrainian).

[12] Andriychuk M. I. New solutions to synthesis problem of plane array with hexagonal geometry. 2014 XIXth International Seminar/Workshop on Direct and Inverse Problems of Electromagnetic and Acoustic Wave Theory (DIPED). 67-71 (2014).

[13] Bahrah L. D., Kremeneckij S. D. Sintez izluchajushhih sistem (teorija i metody rascheta). Moskva, Sovetskoe radio (1974), (in Russian). 


\title{
Синтез двовимірних антенних ґраток методом узагальненого розділення змінних
}

\author{
Ярошко С. М. ${ }^{1}$, Ярошко С. А. ${ }^{2}$ \\ ${ }^{1}$ Націоналъний університет “Лъвівсъка політехніка", \\ вул. С. Бандери, 12, Лъвів, 79013, Україна \\ 2 Лъвівсъкий національний університет імені Івана Франка, \\ вул. Університетсъка, 1, Лъвів, 79000, Україна
}

\begin{abstract}
Метод узагальненого розділення змінних застосовано для розв'язування задач синтезу двовимірних антенних граток заданої форми за заданими амплітудними діаграмами напрямленості. Наближений розв'язок шукають у вигляді суми доданків із розділеними індексами. Такі доданки знаходять послідовно, по одному на кожному кроці методу з умови мінімуму відповідного функціонала. Задачу мінімізації зведено до задачі розв'язування нелінійної системи алгебричних рівнянь. Загальні підходи методу конкретизовано для випадків плоских прямокутних, плоских гексагональних і циліндричних граток. Належну увагу приділено побудові ефективних обчислювальних процедур. Наведено числові результати розв'язування декількох модельних задач. Показано, що метод дає змогу отримати задовільний з практичного погляду наближений розв'язок за два-три кроки.
\end{abstract}

Ключові слова: розділення змінних, синтез антенних ґраток, амплітудна діаграма напрямленості, нелінійна обернена задача.

2000 MSC: $65 \mathrm{C} 20$

УДК: $519.677,004.42$ 